\title{
Short-Term Wave Forecasting for Real-Time Control of Wave Energy Converters
}

\author{
Francesco Fusco and John V. Ringwood
}

\begin{abstract}
Real-time control of wave energy converters requires knowledge of future incident wave elevation in order to approach optimal efficiency of wave energy extraction. We present an approach where the wave elevation is treated as a time series and it is predicted only from its past history. A comparison of a range of forecasting methodologies on real wave observations from two different locations shows how the relatively simple linear autoregressive model, which implicitly models the cyclical behavior of waves, can offer very accurate predictions of swell waves for up to two wave periods into the future.
\end{abstract}

Index Terms-Time series, wave energy, wave forecasting.

\section{INTRODUCTION}

$\mathbf{T}$ HE energy conversion in most wave energy converters (WECs) is based either on relative oscillation between bodies or on oscillating pressure distributions within fixed or moving chambers. Oscillators generally have pronounced resonances, which enable efficient power absorption only over a restricted range of frequencies. In order, however, to cope with the variations of wave spectra, a control system can be designed to alter the oscillator dynamics such that the efficient energy conversion occurs over a wide range of wave conditions [1].

The control approach, in the early stages of wave energy conversion, consisted of frequency domain relationships regulating the dynamics of the system to be tuned for maximum energy absorption at different peak frequencies corresponding to different incoming wave spectra [1], [2]. Although being an advantageous approach for real sea spectra, frequency domain techniques do not generally allow real-time control on a wave-bywave basis, which can significantly raise the device productivity and, therefore, its economical viability. Real-time optimal control can be directly derived from the aforementioned optimal frequency relationships [1], [2]. The main difficulties arise from the fact that the transformation into the time domain results in noncausal transfer functions, so that the conditions for optimal power absorption can be realized only if future motion of the device, or of the future incident wave profile, are known [1]-[3].

The problem of short-term wave prediction, for some seconds into the future, of the actual wave elevation profile at a specific point of the sea surface, is central, therefore, to the more general

Manuscript received December 17, 2009; revised February 22, 2010; accepted March 29, 2010. Date of publication April 19, 2010; date of current version June 23, 2010. This work was supported by the Irish Research Council of Science, Engineering, and Technologies (IRCSET) under the Embark Initiative.

The authors are with the Department of Electronic Engineering, National University of Ireland Maynooth, Maynooth, Ireland (e-mail: francesco@eeng. nuim.ie; fusco@eeng.nuim.ie).

Color versions of one or more of the figures in this paper are available online at http://ieeexplore.ieee.org.

Digital Object Identifier 10.1109/TSTE.2010.2047414

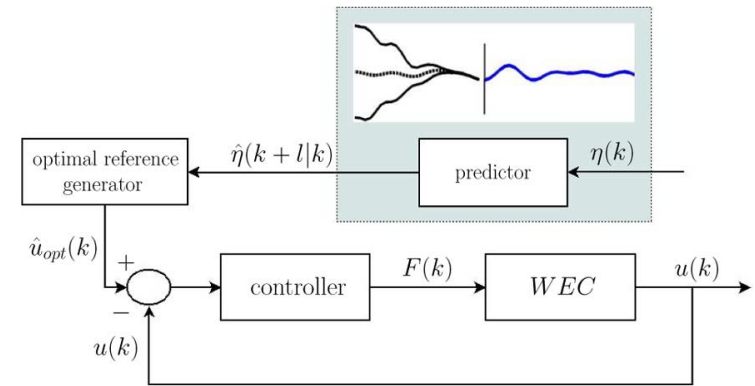

Fig. 1. Wave predictions are required in order to generate an optimal reference for a generic time-domain control of a WEC.

issue of time domain control of WECs. As an example, Fig. 1 illustrates a possible digital control scheme for a generic oscillating body, where the controlled variable is its oscillation velocity $u(k)$ and the control action is performed through a control force $F(k)$. The estimate of the optimal reference $\hat{u}_{\text {opt }}(k)$ is computed by an algorithm that requires future values $\hat{\eta}(k+l \mid k)$ of the incident wave elevation $\eta(k)$. Depending on the operating principle of the specific device, as well as the control strategy adopted and the available instrumentation, the involved quantities may vary, but the logic can always be described by a framework such as that proposed in Fig. 1. In particular, the quantity to be predicted may be the wave excitation force or the oscillation velocity for an oscillating body, the air pressure inside the chamber of an oscillating water column, and they all depend, ultimately, on the incident wave elevation.

The main approach followed in the literature is based on a spatial prediction of the wave elevation, as in Fig. 2(b), meaning that the wave field at a certain location is reconstructed from one or more observations at nearby locations [4]-[7]. The forecasting model, in this situation, requires an array of spatial measurements and can become very complex, because it has to take into account the possible multidirectionality of waves [5], the presence of radiated and diffracted waves [8], and eventual nonlinearities in the waves propagation (refer to [9] for a more detailed literature survey).

The solution proposed in this paper is the prediction of the wave elevation based only on its past history at the same point of the sea surface, as in Fig. 2(a). Such an alternative approach certainly introduces significant advantages with respect to the spatial prediction, in terms of complexity of the models (multidirectionality and radiation do not need to be considered unless the device itself requires it, e.g., directional WECs) and on the amount of instrumentation required (measurements at only one point are required). Some preliminary work, following this purely time series approach, may be found in [1] and [10], where autoregressive (AR) models are proposed for prediction of future oscillation velocity of a generic device, and in [11], where 


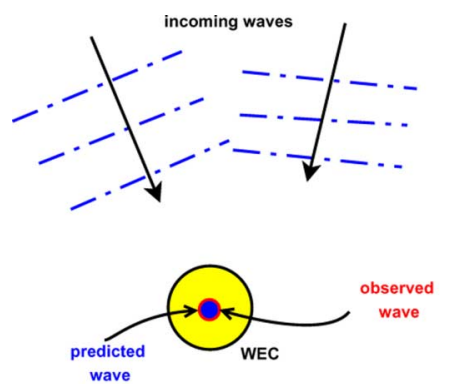

(a)

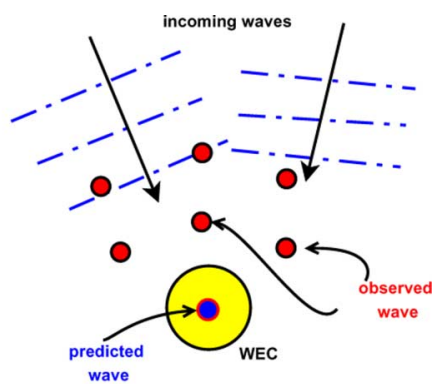

(b)
Fig. 2. Two main approaches to wave forecasting. (a) Prediction based only on local single-point measurements. (b) Prediction based on reconstruction of wave field from array of distant measurements.

AR and hybrid Kautz/AR models are utilized to predict the wave elevation time series. Both of them only present results with data numerically generated from standard wave spectra.

This paper presents a variety of forecasting models and validates them against real observations, following the preliminary results presented in [12] and [13]. The real data available is first analyzed in Section II. The forecasting models are then presented in Section III and a methodology to evaluate the confidence intervals of the predictions is outlined in Section IV. Results are finally discussed in Section V.

\section{Available Data}

The data utilized for this study comes from two locations:

1) The Irish Marine Institute provided real observations from a data buoy located in Galway Bay, on the West Coast of Ireland, at approximately $53^{\circ} 13^{\prime} \mathrm{N}, 9^{\circ} 18^{\prime} \mathrm{W}$ (water depth nearly $20 \mathrm{~m}$ ). Data consist of 20 -min records sets for each hour, collected at a sampling frequency of $16.08 \mathrm{rad} / \mathrm{s}$ $(2.56 \mathrm{~Hz})$. The location is sheltered from the Atlantic Ocean so that the wave height magnitude, in general, is relatively small.

2) Wave elevation time series are also available from the Atlantic Ocean at the Pico Island, in the Azores Archipelago, at approximately $38^{\circ} 33^{\prime} \mathrm{N}, 28^{\circ} 34^{\prime} \mathrm{W}$ (water depth around $40 \mathrm{~m}$ ). The Pico data are collected in the form of two contiguous 30-min record sets for each hour, with a sampling frequency of $8.04 \mathrm{rad} / \mathrm{s}(1.28 \mathrm{~Hz})$ [14]-[16]

The climate at the Galway Bay site is dominated by relatively low energy sea states (significant wave height less than $2 \mathrm{~m}$ ) which, most of the time, have a broad spectral distribution with no clear energy peak, due to the superposition of low-frequency swell(s) and high-frequency wind waves of similar energy content. Wave systems off the coast of Pico, on the other hand, usually have a more defined low-frequency peak (around $0.7 \mathrm{rad} / \mathrm{s}$ ) and their significant wave height ranges, usually, from 1 to $5 \mathrm{~m}$. A more detailed analysis of the wave data at the two locations can be found in [17].

The wave spectra of three significant data sets at the two locations, shown in Fig. 3, will be utilized to test the forecasting models, in Section V. In particular, one wide-banded and one narrow-banded sea state from each of the two sites is considered. Then, a situation where wind waves predominate is selected from the Galway Bay data and a very high-energy wave system, where the sea bottom slightly affects the wave symmetry (this was analyzed through higher order spectral analysis
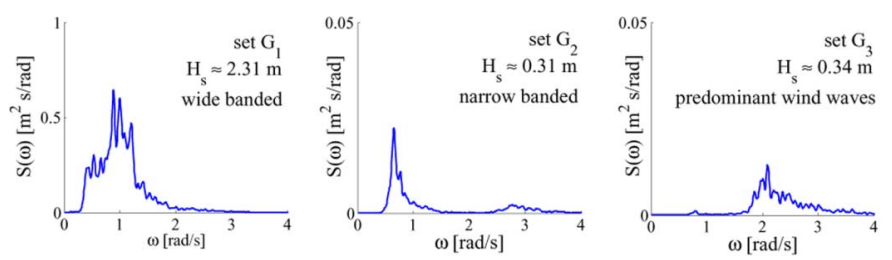

(a)
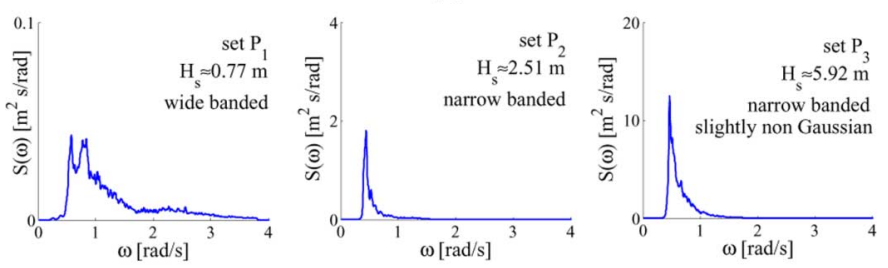

(b)

Fig. 3. Wave spectra of sample data set for the two locations: (a) Galway Bay; (b) Pico Island.

and skewness and kurtosis indices [13]), is chosen from the Pico Island data.

One main characteristic that emerges from the wave data analysis, which is well known, is that a significant portion of the wave energy is usually concentrated at low frequencies. In contrast, high frequencies dominate the spectrum only for very low energy sea states, which are of diminished interest for wave energy conversion. It is also known that low-frequency waves (the swell) are more regular and less affected by nonlinearities (which can be verified by means of the Bispectrum [13], [17], [18]). It is reasonable, therefore, to low-pass filter the wave elevation and focus the prediction only on the low frequencies, which, intuitively, might improve the accuracy of the forecasts and the length of the forecasting horizon.

A quantification of the possible benefits of low-pass filtering on the prediction can be obtained by measuring the predictability of the wave elevation time series. It is argued that for predictability analysis, it is not necessary to design any predictors; we just have to know how much information about future signal values can be obtained from the past [19]. A simpler measure of predictability than the very general approach proposed in [19] and [20] (based on the mutual information notion) will be adopted here, as previously proposed in [13]. It assumes that a linear relationship exists between the future values of the wave elevation and its past values. A predictability index $R^{2}(l)$ is estimated as

$$
R^{2}(l) \triangleq \frac{E\left\{\hat{\eta}(k+l \mid k)^{2}\right\}}{E\left\{\eta(k)^{2}\right\}}
$$

where $\eta(k)$ is the wave elevation, supposed to have a zero mean, $E\{\cdot\}$ is the expectation operator, and $\hat{\eta}(k+l \mid k)$ is the optimal $l$-step ahead prediction. A very efficient algorithm for the estimation of $R(k)^{2}$, which is utilized in this study, was proposed in [21].

In Fig. 4, it is shown that the improvement of predictability of the wave data detailed in Fig. 3, when only low-frequency components are considered, is quite significant, particularly in the case of narrow-banded sea states. The suggestion that it is reasonable to focus the wave forecasting algorithms only on the low-frequency waves is, therefore, numerically confirmed. Note that the choice of the cut-off frequencies $\omega_{c}$ is based on visual inspection of the spectral shape of each data set, such that the 

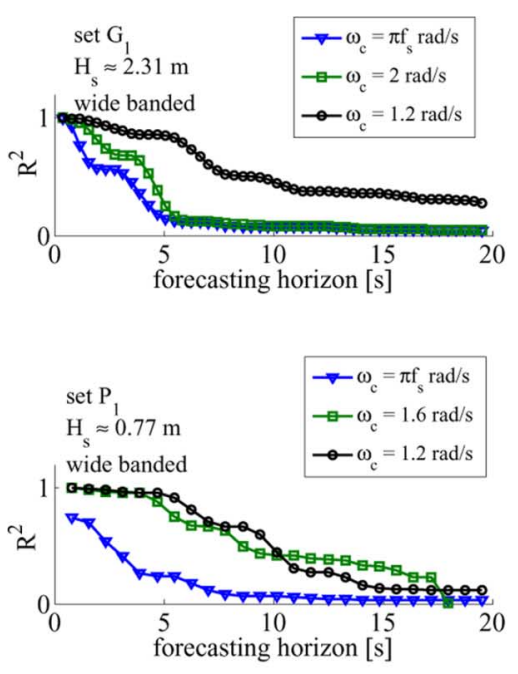

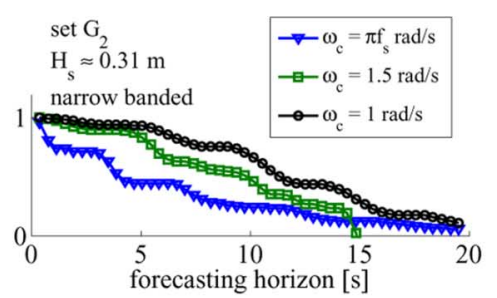

(a)

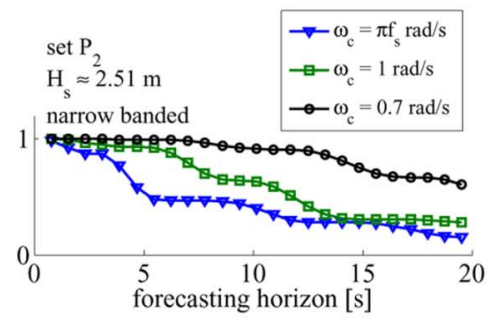

(b)
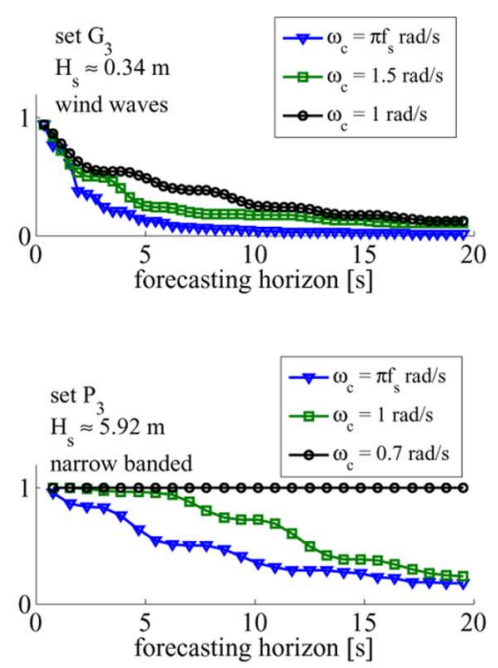

Fig. 4. Predictability of wave elevation time series, low-pass filtered with different cut-off frequencies $\omega_{c}$ : (a) Galway Bay; (b) Pico Island.

amount of energy discarded is not significant (apart from the data set $G_{3}$, where the wind waves dominate the spectrum). The cut-off frequency will ultimately represent a significant parameter at the design stage of any specific wave prediction algorithm and it will be determined as a compromise between improvement in the prediction accuracy and amount of discarded wave components/energy.

\section{FORECASTING MODELS}

\section{A. Cyclical Models}

The most straightforward forecasting model is a cyclical model, where the wave elevation $\eta(k)$ is expressed as a superposition of a number $m$ of linear harmonic components

$$
\eta(k)=\sum_{i=1}^{m} \alpha_{i} \cos \left(\omega_{i} k\right)+\beta_{i} \sin \left(\omega_{i} k\right)+\zeta(k)
$$

An error term $\zeta(k)$ has been introduced, while the phase and amplitude information for each harmonic component is contained in the parameters $\alpha_{i}$ and $\beta_{i}$ [12], [13].

The model in (2) is completely characterized by the parameters $\alpha_{i}, \beta_{i}$ and by the frequencies $\omega_{i}$. It could be fitted to the data through a nonlinear estimation procedure (the model is nonlinear in the frequencies) and utilized to predict the future wave elevation. It needs, however, to be adapted to the time variations of the wave spectrum (amplitudes, phases, and frequencies are nonconstant), so that a first solution has been considered [13], where the frequencies are kept constant and the model becomes perfectly linear in the parameters $\alpha_{i}, \beta_{i}$.

The choice of the frequencies is a crucial one. In [13], it was pointed out how, while the range of frequencies is easy to determine, the distribution of the $\omega_{i}$ inside this range is more problematic. A robust choice would be a constant spacing over all the range, with the spacing as small as possible in order to give an accurate coverage of the spectrum (appropriate constant frequency intervals are also discussed in [22]). Alternative nonhomogeneous solutions may be considered, for example, with the frequencies more densely distributed around the energy peaks. Such more efficient solutions, however, would be unreliable, in the context of a model with fixed frequencies, due to the time variations of the energy distribution in wave spectra (which can be verified through the Wavelet transform [17], [22]).

Once the frequencies are determined, a model for the amplitude variations has to be chosen. Initially, the cyclical structural model proposed by Harvey [23] was adopted

$$
\eta(k)=\sum_{i=1}^{m} \psi_{i}(k)+\zeta(k)
$$

and

$$
\begin{aligned}
{\left[\begin{array}{c}
\psi_{i}(k+1) \\
\psi_{i}^{*}(k+1)
\end{array}\right]=} & {\left[\begin{array}{cc}
\cos \left(\omega_{i} T_{s}\right) & \sin \left(\omega_{i} T_{s}\right) \\
-\sin \left(\omega_{i} T_{s}\right) & \cos \left(\omega_{i} T_{s}\right)
\end{array}\right]\left[\begin{array}{c}
\psi_{i}(k) \\
\psi_{i}^{*}(k)
\end{array}\right] } \\
& +\left[\begin{array}{c}
w_{i}(k) \\
w_{i}^{*}(k)
\end{array}\right], \quad i=1, \ldots m
\end{aligned}
$$

where $T_{s}$ is the sampling time, vectors $\left[\psi_{i}(k) \psi_{i}^{*}(k)\right]^{T}$ model the $m$ cyclical components, and it can be verified that $\psi_{i}(0)=\alpha_{i}$ and $\psi_{i}^{*}(0)=\beta_{i}$. The Gaussian white disturbance $\left[\begin{array}{ll}w_{i}(k) & w_{i}^{*}(k)\end{array}\right]^{T}$ models the amplitude and phases variation. In [12] and [13], an alternative stochastic model for the amplitudes $\alpha_{i}(k)$ and $\beta_{i}(k)$ was proposed: the dynamic harmonic regression (DHR) model [24]. However, the higher complexity required (four state variables for each harmonic against two of the Harvey's model) was not justified by an improvement of the modeling ability, so the DHR model it is not further considered.

From (3) and (4), the following state space form is derived:

$$
\begin{aligned}
\mathbf{x}(k+1) & =\mathbf{A x}(k)+\mathbf{w}(k) \\
\eta(k) & =\mathbf{C x}(k)+\zeta(k)
\end{aligned}
$$

where

$$
\begin{aligned}
& \mathbf{x}(k) \triangleq\left[\psi_{1}(k) \psi_{1}^{*}(k) \ldots \psi_{m}(k) \psi_{m}^{*}(k)\right]^{T} \in \mathbb{R}^{2 m \times 1} \\
& \mathbf{w}(k) \triangleq\left[w_{1}(k) w_{1}^{*}(k) \ldots w_{m}(k) w_{m}^{*}(k)\right]^{T} \in \mathbb{R}^{2 m \times 1} \\
& \mathbf{A} \triangleq \operatorname{diag}\left\{\left[\begin{array}{cc}
\cos \left(\omega_{i} T_{s}\right) & \sin \left(\omega_{i} T_{s}\right) \\
-\sin \left(\omega_{i} T_{s}\right) & \cos \left(\omega_{i} T_{s}\right)
\end{array}\right]\right\} \\
& \in \mathbb{R}^{2 m \times 2 m} \\
& \mathbf{C} \triangleq\left[\begin{array}{lllllll}
1 & 0 & 1 & 0 & \ldots & 1 & 0
\end{array}\right] \in \mathbb{R}^{1 \times 2 m} .
\end{aligned}
$$

The state space form is suited to the application of the Kalman filter for recursive online adaptation. The initialization is pro- 
vided by regular least squares estimates on a number of batch observations and then the Kalman filter is applied online, once a covariance matrix for the state and output disturbances is available. When the estimate of the model's parameters $\hat{\mathbf{x}}(k \mid k)$ is available at any instant $k$, the $l$-step-ahead prediction $\hat{\eta}(k+l \mid k)$ is obtained through the free evolution of the model in (5) as

$$
\hat{\eta}(k+l \mid k)=\mathbf{C A}^{l} \hat{\mathbf{x}}(k \mid k) .
$$

\section{B. Sinusoidal Extrapolation With the Extended Kalman Filter}

A step further from the cyclical models with fixed frequencies would be to consider variable frequencies which are updated online, along with the amplitudes and phases, on the basis of the free surface wave elevation measurements.

We can, therefore, attempt to model the wave elevation as a single cyclical component of the type in (4), but with a timevarying frequency $\omega(k)$

$$
\eta(k)=\psi(k)+\zeta(k)
$$

with

$$
\begin{aligned}
& {\left[\begin{array}{c}
\psi(k+1) \\
\psi^{*}(k+1) \\
\omega(k+1)
\end{array}\right]=\left[\begin{array}{ccc}
\cos \left(\omega(k) T_{s}\right) & \sin \left(\omega(k) T_{s}\right) & 0 \\
-\sin \left(\omega(k) T_{s}\right) & \cos \left(\omega(k) T_{s}\right) & 0 \\
0 & 0 & 1
\end{array}\right]} \\
& \cdot\left[\begin{array}{c}
\psi(k) \\
\psi^{*}(k) \\
\omega(k)
\end{array}\right]+\left[\begin{array}{c}
\varepsilon(k) \\
\varepsilon^{*}(k) \\
\kappa(k)
\end{array}\right]
\end{aligned}
$$

where $\varepsilon(k), \varepsilon^{*}(k)$, and $\zeta(k)$ are random disturbances, $\eta(k)$ is the wave elevation, and a model for the variability of $\omega(k)$ has been introduced, assuming a simple random walk driven by the additional white noise $\kappa(k)$. The model in (12), of course, is nonlinear in $\omega(k)$ and an explicit linear state space structure cannot be formulated.

As a consequence, a linear recursive estimator cannot be directly applied. It is possible, however, to utilize an extension of the Kalman filter to nonlinear models, namely the extended Kalman filter (EKF), assuming that the discrete time step $T_{s}$ is sufficiently small to permit the prediction equations to be approximated by a linearized form, based on the truncation of the Taylor expansion of (12) at the first order [25]. The estimate of the state vector at each time step is, therefore, given by linear recursive equations, while the prediction $\hat{\eta}(k+l \mid k)=\hat{\psi}(k+l \mid k)$ is obtained from the free evolution of the nonlinear model in (12).

The extension to a model with $m>1$ variable frequencies is not straightforward. It was found that a superposition of $m$ models of the type expressed in (12) does not offer any actual advantage [17]. Such an extension is under study, at the moment, and the current study is focused on a single frequency, so that the model can be considered as a sinusoidal extrapolation.

\section{AR Models}

The wave elevation $\eta(k)$ is assumed to be linearly dependent on a number $n$ of its past values, through the parameters $a_{i}$

$$
\eta(k)=\sum_{i=1}^{n} a_{i} \eta(k-i)+\zeta(k)
$$

where a disturbance term $\zeta(k)$ has been included. If an estimate of the parameters at instant $k, \hat{a}_{i}(k)$, is computed and the noise is assumed to be Gaussian and white, the best prediction of the future wave elevation $\hat{\eta}(k+l \mid k)$ at instant $k$ can be derived from (13) as

$$
\hat{\eta}(k+l \mid k)=\sum_{i=1}^{n} \hat{a}_{i}(k) \hat{\eta}(k+l-i \mid k)
$$

where, obviously, $\hat{\eta}(k+l-i \mid k) \equiv \eta(k)$ if $k+l-i \leq k$ (i.e., information already acquired, no need of prediction).

The general shape of the prediction function $\hat{\eta}(k+l \mid k)$ is completely determined by the poles $p_{i}$ of the AR model in (13). In the case of $m / 2$ (when $m$ is even) couples of complex-conjugate poles, $p_{i}$ and $p_{i}^{*}$, [26]

$$
\hat{\eta}(k+l \mid k)=\sum_{i=1}^{m / 2} c_{i}(k)\left|p_{i}\right|^{l} \sin \left(\angle p_{i} k+\varphi_{i}(k)\right)
$$

where the coefficients $c_{i}(k)$ depend on the last $n$ observations $\eta(k), \eta(k-1), \ldots \eta(k-n+1)$. Thus, an AR model with only complex-conjugate poles is implicitly a cyclical model, where the frequencies are related to the phase $\angle p_{i}$ of each pole and the amplitudes and phases of the harmonic components are related to the last $n$ measurements of each time instant $k$, so that they adapt to the observations.

The AR coefficients $a_{i}$ are estimated from a number $N$ of batch observations through the minimization of a multistep ahead cost functional, referred to as long-range predictive identification (LPRI) [27]

$$
J_{\mathrm{LPRI}}=\sum_{k=1}^{N} \sum_{j=1}^{N_{l}}[\eta(k)-\hat{\eta}(k \mid k-j)]^{2}
$$

where $N_{l}$ is the forecasting horizon over which the AR model is to be optimized. The function $J_{\text {LPRI }}$ is minimized with a standard algorithm for nonlinear least squares problems, the Gauss-Newton algorithm, initialized with the estimates from regular least squares [27].

Note that, from (15), the implicit frequencies are related to the poles of the AR model, so if the AR parameters are kept constant, the frequencies will be constant as well. An adaptivity mechanism based on the LPRI function (16) could be implemented, as proposed in [27]. However, as will also be shown in the results of Section V, a static AR model maintains its accuracy for a long time after being estimated (more than 2 hours), in spite of spectral variations (for more details refer to [17]). Adaptive AR models, therefore, are not a priority and are not considered in the present work.

\section{Neural Networks}

In spite of the nonlinear modeling capability, neural networks have the great disadvantage of offering a model completely enclosed in a black box, where inherent characteristics cannot be analyzed by inspection or analytical calculation. So, whereas in the cyclical and AR models an analysis of the estimated parameters and frequencies and their variations in an adaptive structure can provide indications about the real process behavior and its main characteristics, this would not be possible with neural networks. 
For the problem under study, a nonlinear relationship is created through a multilayer perceptron [28]

$$
\eta(k)=\mathbf{N N}(\eta(k-1), \eta(k-2), \ldots \eta(k-n)) .
$$

Only structures with one linear output neuron and two hidden layers, consisting of a number of nonlinear neurons, varying between three and seven each, were considered. Several orders of regression $n$ were also considered. The model is trained using the Levenberg-Marquard algorithm [29] on a set of batch data and utilized for multistep-ahead prediction.

The structure in (17) is, of course, not the only possibility and many others could be considered. For example, a priori knowledge about the process (harmonic nature) may be considered as follows:

$$
\eta(k)=\mathbf{N N}\left(\cos \left(\omega_{1} T_{s} k+\varphi_{1}\right), \ldots \cos \left(\omega_{n} T_{s} k+\varphi_{n}\right)\right)
$$

though such an input structure would retain some of the limitations of cyclical models with fixed frequencies.

\section{E. Other Possibilities}

Other possible wave forecasting models were analyzed, but were found unsuitable and therefore discarded (refer to [17] for a detailed discussion).

A candidate alternative technique is Gaussian Processes (GPs), whose basic idea is to place a Gaussian prior directly on the space of functions [30] underlying the data, without assuming any particular function parametrization but only specifying the prior's mean and covariance. In order to model the cyclical characteristics of the sea, the harmonic frequencies of the covariance function have to be assigned permanently in the initial estimation of the model. This leads to the issue, concerning the choice of frequencies, that emerged from the discussion of cyclical models with fixed frequencies (see Section III-A).

Also, the introduction of a moving average (MA) term in the AR model, giving an ARMA model, has been analyzed and it was found that no real change in the forecasting function's shape, with respect to AR models, can be obtained, so that no significant improvement in the results can be expected. ARMA models can, however, be utilized in order to obtain more parsimonious forecasting models, but this is not the focus of the study and it is not considered here.

Particle filters [31] may be considered for the online state estimation of the nonlinear model (12) and can deal directly with nonlinear models. However, while they do not assume a Gaussian distribution of the state variable, and therefore may give better estimation results than the EKF, they still only allow a single frequency to be tracked, and would, therefore, be subject to the main performance limitation as the EKF.

\section{CONFIDENCE INTERVALS}

The wave elevation predictions alone, as computed by any of the models presented, do not give sufficiently complete information about the future of the signal, as they are inevitably affected by an error. It is a fundamental need to have an indication about the extent of this error and about the confidence that we can put in the forecasts computed by the prediction algorithm. If the $l$-step ahead prediction error is Gaussian

$$
\hat{e}(k+l \mid k)=\eta(k+l)-\hat{\eta}(k+l \mid k) \approx \aleph\left(0, \sigma_{l}^{2}\right)
$$

then the variance $\sigma_{l}^{2}$ is all we need in order to define its probability distribution. We can assume that the error is contained within a confidence interval, with probability $\delta$, as follows:

$$
-z_{(\delta / 2)} \leq \hat{e}(k+l \mid k) \leq+z_{(\delta / 2)} .
$$

In $(20), z_{(\delta / 2)}$ is the value of the probability distribution such that

$$
P\left\{-z_{(\delta / 2)} \leq \hat{e}(k+l \mid k) \leq+z_{(\delta / 2)}\right\} \triangleq \int_{-z_{(\delta / 2)}}^{+z_{(\delta / 2)}} p(y) d y=\delta
$$

where the probability density function $p(\cdot)$, with the distribution of the forecasting error considered to be zero-mean Gaussian, assumes the following structure:

$$
p(\hat{e}(k+l \mid k))=\frac{1}{\sqrt{2 \pi} \sigma_{l}} e^{-\left(\hat{e}(k+l \mid k)^{2} / 2 \sigma_{l}^{2}\right)} .
$$

The estimate of the variance $\sigma_{l}^{2}$ could be calculated from the specific model parameters and from the statistics of the parameter estimation algorithm, which is not straightforward and also could be misleading if the model is not sufficiently accurate. A more straightforward alternative, however, is adopted, where the estimate of the variance of the forecasting error is based purely on the past history of the prediction errors

$$
\hat{\sigma}_{l}^{2}=\frac{1}{N-1} \sum_{k=1}^{N} \hat{e}(k+l \mid k)^{2}
$$

where $N$ is the number of past observations available.

The estimate of $\sigma_{l}^{2}$ can also be recursively updated as soon as new observations become available [32], via

$$
\hat{\sigma}_{l}^{2}(k)=\frac{k-2}{k-1} \hat{\sigma}_{l}(k-1)+\frac{1}{k}[\eta(k)-\hat{\eta}(k \mid k-l)]^{2}, \quad k \geq 2 .
$$

\section{RESULTS}

Each of the data sets of Fig. 3 was split up into training and validation sets. For the Galway Bay data, the training and validation sets consist of two consecutive data sets of 3072 samples (20 min each at a sampling frequency of $16.08 \mathrm{rad} / \mathrm{s}$, or $2.56 \mathrm{~Hz}$ ). In the case of the Pico Island data, because the consecutive data sets are actually contiguous in time, training and validation sets have been chosen as four consecutive segments each $(9216 \mathrm{sam}$ ples per set, meaning $2 \mathrm{~h}$ at a sampling frequency of $8.04 \mathrm{rad} / \mathrm{s}$, or $1.28 \mathrm{~Hz}$ ).

The prediction accuracy is measured with the following goodness-of-fit index, for each forecasting horizon $l$ :

$$
\mathcal{F}(l)=\left(1-\frac{\sqrt{\sum_{k}[\eta(k+l)-\hat{\eta}(k+l \mid k)]^{2}}}{\sqrt{\sum_{k} \eta(k)^{2}}}\right) \cdot 100 .
$$



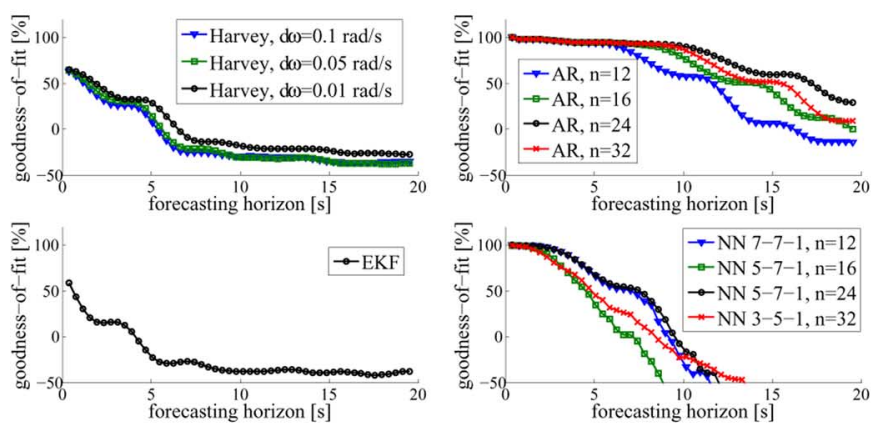

(a)
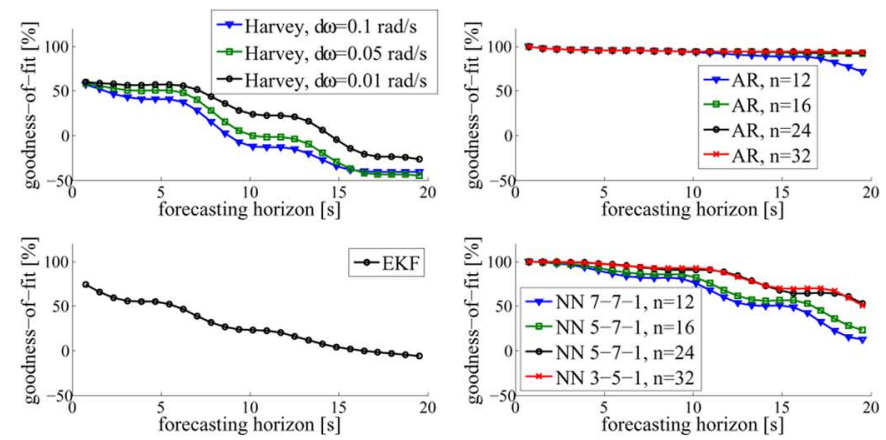

(b)

Fig. 5. Goodness-of-fit $\mathcal{F}(l)$ of different forecasting models on two data sets: (a) Galway Bay set $G_{1}$; (b) Pico Island set $P_{2}$.

Here $\eta(k+l)$ is the wave elevation and $\hat{\eta}(k+l \mid k)$ is its prediction based on the information up to instant $k$. A $100 \%$ value for $\mathcal{F}(l)$ means that the wave elevation time series is perfectly predicted $l$ steps into the future. Note that the quantity $\mathcal{F}(l)$ has a direct correspondence with the variance of the prediction error which, as discussed in Section IV, is utilized to characterize the confidence interval of the forecasts. In particular, if $\hat{\sigma}_{l}^{2}$ is an estimate of the variance of the $l$-step ahead prediction error, then

$$
\hat{\sigma}_{l}^{2}=\left(1-\frac{\mathcal{F}(l)}{100}\right)^{2} \sum_{k} \eta(k)^{2} .
$$

The focus is on the prediction of the low-frequency components, which contain the most energy, as discussed in Section II. Ideal zero-phase low-pass filters are approximated with offline forward and backward filtering through type $I$ Chebyshev filters (order 15, maximum error in the passband $10^{-3}$ ) discretized with the bilinear transform. The cut-off frequency $\omega_{c}$ has been chosen according to the specific shape of the wave spectrum (Fig. 3). Fig. 5 shows the prediction accuracy of different configurations of the models outlined in Section III, for two wave systems, over a forecasting horizon of about $20 \mathrm{~s}$ (50 samples for the Galway data and 25 samples for the Pico data).

The first thing that emerges from Fig. 5 is that all the selected forecasting models perform better for the narrow-banded sea state $P_{2}$, as shown in Fig. 5(b), than for the broader-banded $G_{1}$, shown in Fig. 5(a), and this was expected after the predictability analysis carried on the wave data, shown in Fig. 4. In particular, the Harvey cyclical model and the sinusoidal extrapolation through the EKF are the most dramatically affected by the bandwidth of the wave signal and their $\mathcal{F}(l)$ for the data set $G_{1}$ dies out relatively quickly, going below $0 \%$ after only $5 \mathrm{~s}$. Note also that a cyclical model with a single variable frequency offers an accuracy comparable with the cyclical models with many fixed frequencies, and at the same time represents a much more efficient approach (three system states compared to hundreds for the Harvey model).

AR models offer much more accurate predictions further into the future than the cyclical models for all the sea states. In the case of $P_{2}$ [Fig. 5(b)], an accuracy of $\mathcal{F}(l)>90 \%$ is maintained for more than $20 \mathrm{~s}$ into the future, about two wave periods of a wave with period $10.47 \mathrm{~s}$ (corresponding to a peak frequency of $0.6 \mathrm{rad} / \mathrm{s}$, as from Fig. 3). AR model performance is still affected by the bandwidth of the sea state, but relatively accurate predictions, $\mathcal{F}(l)>90 \%$ for more than $10 \mathrm{~s}$ in the future, are still obtained for the set $G_{1}$, although higher order models, e.g., $n=24,32$, are required.

For the neural networks, the results of some of the more promising configurations show a similar accuracy to AR models for the case of the narrow-banded sea state $P_{2}$, while the prediction diverges fairly quickly in the case of the data set $G_{1}$. The search of possible network configurations was not exhaustive, but was broad enough to conclude that neural networks are not able to offer any significant improvement in the short-term prediction of the wave elevation, with respect to simple AR models, such to justify the higher complexity and the lack of any physical meaning. Note that high-energy sea states with a well-defined low-frequency swell like $P_{2}$ present relatively weak nonlinearities (from analysis of the Bispectrum, and of skewness and kurtosis indices [17]) unless shallow water conditions apply (which is not the case here).

More detailed results over the six sea states considered in this study are shown in Table I, regarding the Galway Bay wave data, and in Table II, for the data from Pico Island. Negative values of $\mathcal{F}(l)$ were omitted, as they mean that the prediction diverges. The results regarding the sea states $G_{2}$ (narrow-banded) and $P_{1}$ (broad-banded) do not add any significant information to the discussion outlined so far, focused on the sea states $G_{1}$ and $P_{2}$ and on Fig. 5. A further interesting point, however, concerns the situation for the sea state dominated by wind waves $G_{3}$. In this case, the neural networks retain a good accuracy in the shortterm, with $\mathcal{F}(l)$ around $90 \%$ for $l=10$, outperforming the AR models, which are significantly affected by the nonlinearities in the wave system (detectable through the Bispectrum, refer to [17] for a detailed analysis).

The detail of the prediction $\hat{\eta}(k+l \mid k)$ with an AR model on the data set $P_{2}$, at a specific instant $k=55$ and for a forecasting horizon $l=1$ to 50 samples (nearly 39 s), is shown in Fig. 6, along with the $90 \%$ confidence interval, estimated according to the methodology described in Section IV. In Fig. 7, the comparison between the real wave elevation and the 25 -step-ahead prediction $\hat{\eta}(k+25 \mid k)$ is shown, computed with an AR model of order $n=24$, along with the corresponding 90\% confidence interval.

\section{CONCLUSION}

This study was focused on the problem of short-term wave prediction, which is a central topic in the wave energy field, in order to improve the economic viability of a WEC. As opposed 
TABLE I

Goodness-of-Fit $\mathcal{F}(l)$ OF Different ForeCASting Models on the GalWay Bay Data Sets of Fig. 3(a)

\begin{tabular}{|c|c|c|c|c|c|c|c|c|c|c|}
\hline & & $G_{1}$ & $c=1.2$ & $d / s$ & $G_{2}$ & $\nu_{c}=1 r$ & $d / s$ & $G_{3}$ & $\omega_{c}=1 r$ & \\
\hline & model & $l=10$ & $l=25$ & $l=50$ & $l=10$ & $l=25$ & $l=50$ & $l=10$ & $l=25$ & $l=50$ \\
\hline & $d \omega=0.1$ & $25.6 \%$ & - & - & $30.6 \%$ & - & - & - & - & \\
\hline Harvey & $d \omega=0.05$ & $30.7 \%$ & - & - & $38.3 \%$ & - & - & - & - & \\
\hline & $d \omega=0$ & $32.4 \%$ & - & - & $47.6 \%$ & - & - & - & - & \\
\hline EKF & - & $5.4 \%$ & - & - & $46.0 \%$ & - & - & $25.3 \%$ & - & \\
\hline & $=12$ & $94.9 \%$ & $58.2 \%$ & & $98.1 \%$ & $87.1 \%$ & $37.2 \%$ & $72.2 \%$ & $57.6 \%$ & $12.2 \%$ \\
\hline $\mathrm{AR}$ & 24 & $94.9 \%$ & $88.3 \%$ & $9.1 \%$ & $98.1 \%$ & & $66.3 \%$ & $72.2 \%$ & $68.8 \%$ & $28.4 \%$ \\
\hline & $=32$ & $94.9 \%$ & $91.4 \%$ & $29.3 \%$ & $98.1 \%$ & $94.6 \%$ & $69.9 \%$ & $72.2 \%$ & $68.8 \%$ & $41.8 \%$ \\
\hline & $7-7-1, n$ & $84.3 \%$ & - & - & $89.4 \%$ & $48.9 \%$ & - & $86.1 \%$ & $24.1 \%$ & \\
\hline NN & $5-7-1, n=2$ & $84.4 \%$ & - & - & $91.1 \%$ & $59.6 \%$ & - & $89.3 \%$ & $39.8 \%$ & \\
\hline & $3-5-1, n=32$ & $68.0 \%$ & - & - & $85.3 \%$ & $30.1 \%$ & - & $93.8 \%$ & $20.1 \%$ & \\
\hline
\end{tabular}

TABLE II

Goodness-of-Fit $\mathcal{F}(l)$ OF Different Forecasting Models on the Pico IsLAND Data SeTS OF Fig. 3(b)

\begin{tabular}{|c|c|c|c|c|c|c|c|c|c|c|}
\hline & & & $g_{c}=1 r$ & $d / s$ & $P_{2}$, & $c=0.7$ & $d / s$ & & $c=0.7$ & $d / s$ \\
\hline & model & $l=5$ & $l=12$ & $l=25$ & $l=5$ & $l=12$ & $l=25$ & $l=5$ & $l=12$ & $l=25$ \\
\hline & $d \omega=0.1$ & $15.0 \%$ & - & - & $41.2 \%$ & & - & $38.0 \%$ & & \\
\hline Harvey & $\omega=0.05$ & $20.4 \%$ & - & - & & $5.6 \%$ & - & $43.5 \%$ & $0.3 \%$ & \\
\hline & & 28.8 & - & - & 56.7 & $6 \%$ & - & $53.1 \%$ & $27.5 \%$ & \\
\hline EKF & - & $8.3 \%$ & - & - & $55.2 \%$ & $24.2 \%$ & - & $56.3 \%$ & $20.8 \%$ & \\
\hline & $=12$ & $96.9 \%$ & $69.6 \%$ & - & 95. & 94. & $71.6 \%$ & $98.7 \%$ & $96.4 \%$ & $81.1 \%$ \\
\hline AR & $=24$ & $99.0 \%$ & $95.7 \%$ & $42.9 \%$ & $95.9 \%$ & 94. & $92.6 \%$ & & $7 \%$ & $93.4 \%$ \\
\hline & $=32$ & $99.0 \%$ & $96.5 \%$ & $52.3 \%$ & $95.9 \%$ & 94. & & $98.7 \%$ & $96.7 \%$ & $93.7 \%$ \\
\hline & & & $20.9 \%$ & & & & & & & $39.9 \%$ \\
\hline NN & $5-7-1, n=24$ & $73.8 \%$ & 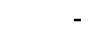 & - & $98.9 \%$ & $90.4 \%$ & $52.8 \%$ & $99.7 \%$ & $95.5 \%$ & $71.8 \%$ \\
\hline & $3-5-1, n=32$ & $74.7 \%$ & - & - & $98.7 \%$ & $92.5 \%$ & $50.6 \%$ & $97.3 \%$ & $91.5 \%$ & $48.8 \%$ \\
\hline
\end{tabular}

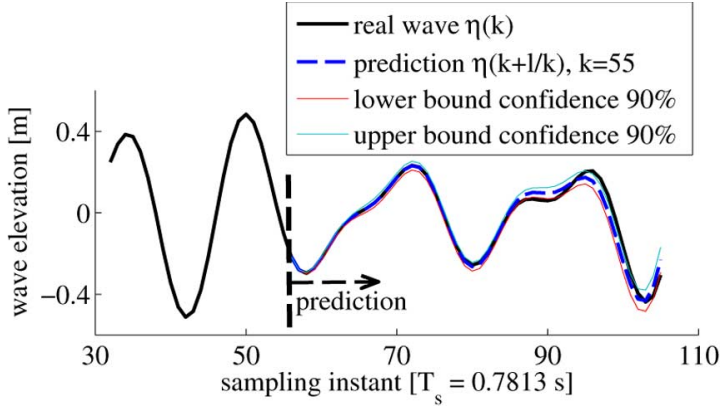

Fig. 6. Confidence interval and predictions $\hat{\eta}(k+l \mid k)$, for $l=1$ to $l=50$, at a specific time instant $k$, calculated with an AR model of order $n=24$ on the data set $P_{2}$, filtered with cut-off frequency $\omega_{c}=0.7 \mathrm{rad} / \mathrm{s}$.

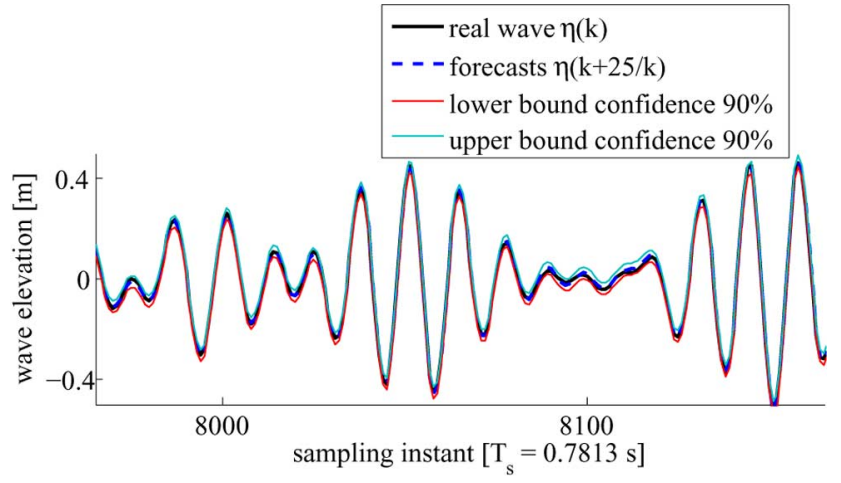

Fig. 7. Confidence interval and 25-step-ahead predictions $\hat{\eta}(k+25 \mid k)$, for some $k$, calculated with an AR model of order $n=24$ on the data set $P_{2}$, filtered with cut-off frequency $\omega_{c}=0.7 \mathrm{rad} / \mathrm{s}$.

to the classic reconstruction of the wave field from distant measurements, an approach was proposed where the wave elevation is treated as a univariate time series and it is forecasted only on the basis of its past history.
Results on real wave elevation data from Galway Bay and Pico Island showed how a relatively simple AR model, which implicitly models the cyclical behavior of the waves, can offer a very accurate prediction of the low-frequency swell waves for up to two typical wave periods into the future. It was also shown that no real benefit can be expected in using nonlinear forecasting models, such as neural networks, unless highly nonlinear sea states are encountered. In deep water locations, however, nonlinearities due to interactions between different wave components only occur for very low-energy wind wave systems, which are of poor interest in a wave energy context (an analysis of nonlinearities in waves is provided in [17]).

Concerning the use of the wave prediction algorithms in realtime WEC control, the real-time filter implementation needs to be given further attention, since a zero-phase filter was assumed in the current analysis. In this case, we feel justified in the choice, since the focus is on looking at achievable prediction. In addition, as mentioned in Section I, the controller of a WEC may require the prediction of an effect of the wave elevation on the device, e.g., the wave excitation force, which is effectively a low-pass filtered version of the wave elevation, with the filtering provided by the device itself.

Further work is also underway in order to quantify the real prediction needs of the WEC control system, in terms of accuracy, forecasting horizon and also general characteristics of the prediction error (e.g., statistical or frequency distribution). This in fact is fundamental in order to compare and to judge more adequately the different forecasting models.

\section{ACKNOWLEDGMENT}

The authors would like to thank the Irish Marine Institute for providing the Galway Bay data. The data from 
Pico Island is available by courtesy of the Centre of Climate, Meteorology, and Global Change of the University of the Azores, projects CLIMAAT; CLIMARCOST; MacSIMAR (FEDER-PIC_Interreg_IIIB \& MAC; MAC2.3/A3; 03/MAC/2.3/A5; 05/MAC/2.3/A1; MAC/1/AO89).

\section{REFERENCES}

[1] U. A. Korde, "Control system applications in wave energy conversion," in Proc. OCEANS 2000 MTS/IEEE Conf. Exhibition, 2000, vol. 3, pp. $1817-1824$

[2] J. Falnes, Ocean Waves and Oscillating Systems. Cambridge, U.K.: Cambridge Univ. Press, 2002.

[3] J. Falnes, "A review of wave-energy extraction," Marine Structures, vol. 20, pp. 285-201, 2007.

[4] K. Budal and J. Falnes, "The Norwegian wave-power buoy project," in 2nd Int. Symp. Wave Energy Utillization, Trondheim, Norway, Jun. 1982.

[5] M. Belmont, J. Horwood, R. Thurley, and J. Baker, "Filters for linear sea-wave prediction," Ocean Eng., vol. 33, pp. 2332-2351, 2006.

[6] J. Tedd, W. Knapp, P. Frigaard, and J. P. Kofoed, "Turbine control strategy including wave prediction for overtopping wave energy converters," in Proc. CA-OE Workshop, Uppsala, Sweden, 2005.

[7] J. Tedd and P. Frigaard, "Short term wave forecasting, using digital filters, for improved control of wave energy converters," in Proc. Int. Offshore Polar Eng. (ISOPE) Conf., 2007, pp. 388-394.

[8] P. Frigaard and M. Brorsen, "A time-domain method for separating incident and reflected irregular waves," Coastal Eng., vol. 24, pp. 205-215, 1995.

[9] F. Fusco, Forecasting Requirements in the Optimal Control of Wave Energy Converters Dept. of Electronic Eng., NUI Maynooth, Ireland, Tech. Rep. EE/2009/2/JVR, 2009.

[10] U. A. Korde, "Efficient primary energy conversion in irregular waves," Ocean Eng., vol. 26, pp. 625-651, 1999.

[11] M. P. Shoen, J. Hals, and T. Moan, "Wave prediction and fuzzy logic control of wave energy converters in irregular waves," in Proc. 16th Mediterranean Conf. Control and Automation, 2008, pp. 767-772.

[12] F. Fusco and J. Ringwood, "Linear models for short term wave forecasting," in Proc. World Renewable Energy Congress (WRECX), 2008, pp. 1030-1035.

[13] F. Fusco and J. Ringwood, "A study on short-term sea profile prediction for wave energy applications," in Proc. 8th Eur. Wave and Tidal Energy Conf. (EWTEC), 2009, pp. 756-765.

[14] R. Esteves, A. Valente, M. Costa, F. Reis, and E. Azevedo, "Dados direccionais de agitao martima nos aores (projecto climaat-clima e meteorologia dos arquiplagos atlnticos)," 6ts Jornadas Portuguesas de Engenharia Costeira e Portuária, PIANC, Funchal, 2009.

[15] E. Azevedo and M. Rodrigues, "Projectos climaat \& climarcost clima e meteorologia dos arquiplagos atlnticos, clima martimo e costeiro," in Workshop Intenacional Sobre Clima e Recursos Naturais nos Países de Língua Portuguesa (WSCRA08), Ilha do Sol, Cape Verde, 2008.

[16] C. Barrera, E. Azevedo, M. Rueda, M. Gelado, and O. Llinás, "Realtime monitoring network in the macaronesian region as a contribution to the coastal ocean observations panel (COOP)," J. Oper. Oceanography, vol. 1, pp. 59-69, 2008.

[17] F. Fusco, Short-Term Wave Forecasting as a Univariate Time Series Problem Dept. of Electronic Eng., NUI Maynooth, Ireland, Tech. Rep. EE/2009/3/JVR, 2009.

[18] M. K. Ochi, Applied Probability and Stochastic Processes. Hoboken, NJ: Wiley Inter-Science, 1990.

[19] H.-P. Bernhard, "A thight upper bound on the gain of linear and nonlinear predictors for stationary stochastic processes," IEEE Trans. Signal Process., vol. 46, no. 11, pp. 2909-2917, Nov. 1998.

[20] C. Shannon, "The mathematical theory of communication," Bell Syst. Tech. J., no. 27, pp. 379-423, 1948.
[21] X. Hong and S. Billings, "Time series multistep-ahead predictability estimation and ranking," J. Forecasting, vol. 18, pp. 139-149, 1999.

[22] G. Nolan, J. Ringwood, and B. Holmes, "Short term wave energy variability off the west coast of Ireland," in Proc. 7th Eur. Wave and Tidal Energy Conf. (EWTEC), Porto, Portugal, 2007.

[23] A. C. Harvey, Forecasting, Structural Time Series Models and the Kalman Filter. Cambridge, U.K.: Cambridge Univ. Press, 1989.

[24] P. C. Young, D. J. Pedregal, and W. Tych, "Dynamic harmonic regression,” J. Forecasting, vol. 18, pp. 369-394, 1999.

[25] B. Quine, J. Uhlmann, and H. Durrant-Whyte, "Implicit Jacobians for linearised state estimation in nonlinear systems," in Proc. Amer. Control Conf., Seattle, WA, 1995, pp. 1645-1646.

[26] G. E. P. Box, G. M. Jenkins, and G. C. Reinsel, Time Series Analysis: Forecasting and Control. Englewood Cliffs, NJ: Prentice-Hall, 1994.

[27] D. S. Shook, C. Mohtadi, and S. L. Shah, "Identification for long-range predictive control," Proc. Inst. Elect. Eng., D Control Theory and Applications, vol. 138, no. 1, pp. 75-84, 1991.

[28] M. Norgaard, O. Ravn, N. Poulsen, and L. Hansen, Neural Networks for Modelling and Control of Dynamic Systems. New York: Springer, 2000 .

[29] B. M. Wilamowski, S. Iplikci, O. Kaynak, and M. O. Efe, "An algorithm for fast convergence in training neural networks," in Proc. Int. Joint Conf. Neural Networks (IJCNN), Washington, DC, 2001, pp. 1778-1782.

[30] D. J. Mackay, "Introduction to Gaussian processes," in Proc. Neural Networks and Machine Learning Conf., 1997, pp. 133-165.

[31] C. P. Robert and G. Casella, Monte Carlo Statistical Methods. New York: Springer, 2004.

[32] R. Isermann, Fault-Diganosis Systems: An Introduction From Fault Detection to Fault Tolerance. New York: Springer, 2006.

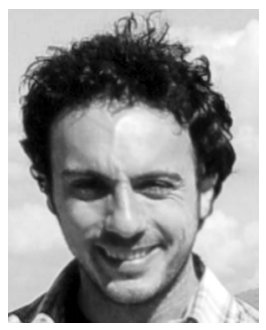

Francesco Fusco received the Bachelors degree in computer science and automation engineering from Universita' Politecnica delle Marche (UNIVPM), Ancona, Italy, in 2006. In December 2008, he received the Masters degree in industrial automation engineering from UNIVPM, with his final thesis, about wave forecasting, carried out at National University of Ireland (NUI), Maynooth, Ireland.

Since January 2009, he is a Ph.D. student as part of the Dynamics and Control Research Group at NUI Maynooth. His research project is focused on the optimization of wave energy converters control systems through prediction of the future incident wave elevation or wave excitation force, and it is funded by the Irish Research Council for the Sciences, Engineering, and Technology (IRCSET).

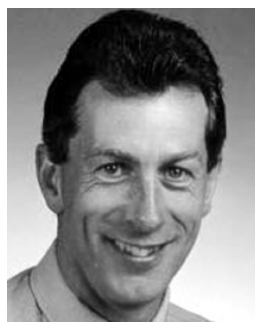

John V. Ringwood received the Diploma in electrical engineering from Dublin Institute of Technology and the Ph.D. degree in control systems from Strathclyde University, Scotland, in 1981 and 1985 , respectively.

$\mathrm{He}$ is currently Professor of Electronic Engineering with the National University of Ireland (NUI), Maynooth, and Associate Dean for Engineering in the Faculty of Science and Engineering. He was Head of the Electronic Engineering Department at NUI Maynooth from 2000 until 2005, developing the Department from a greenfield site. John's research interests cover time series modeling, wave energy, control of plasma processes, and biomedical engineering.

Dr. Ringwood is a Chartered Engineer and a Fellow of the Institution of Engineers of Ireland. 